Original Article

\title{
Ulnar nerve entrapment neuropathy at the elbow: relationship between the electrophysiological findings and neuropathic pain
}

\author{
Gulistan Halac $^{1)^{*}}$, Pinar Topaloglu ${ }^{2)}$, Saliha Demir ${ }^{3)}$, Mehmet Ali Cikrikcioglu4), \\ Hasan Huseyin Karadeli ${ }^{1)}$, Muhammet Emin Ozcan ${ }^{1)}$, Talip Asil ${ }^{1)}$ \\ 1) Department of Neurology, Medical Faculty, Bezmialem Vakıf University: 34093 Fatih Istanbul, \\ Turkey \\ 2) Department of Neurology, Istanbul Medical Faculty, Istanbul University, Turkey \\ 3) Department of Physical Medicine and Rehabilitation, Medical Faculty, Bezmialem Vakif University, \\ Turkey \\ 4) Department of Internal Medicine, Medical Faculty, Bezmialem Vakif University, Turkey
}

\begin{abstract}
Purpose] Ulnar nerve neuropathies are the second most commonly seen entrapment neuropathies of the upper extremities after carpal tunnel syndrome. In this study, we aimed to evaluate pain among ulnar neuropathy patients by the Leeds assessment of neuropathic symptoms and signs pain scale and determine if it correlated with the severity of electrophysiologicalfindings. [Subjects and Methods] We studied 34 patients with clinical and electrophysiological ulnar nerve neuropathies at the elbow. After diagnosis of ulnar neuropathy at the elbow, all patients underwent the Turkish version of the Leeds assessment of neuropathic symptoms and signs pain scale. [Results] The ulnar entrapment neuropathy at the elbow was classified as class-2, class-3, class-4, and class-5 (Padua Distal Ulnar Neuropathy classification) for $15,14,4$, and 1 patient, respectively. No patient included in class-1 was detected. According to Leeds assessment of neuropathic symptoms and signs pain scale, 24 patients scored under 12 points. The number of patients who achieved more than 12 points was 10 . Groups were compared by using the $\chi^{2}$ test, and no difference was detected. There was no correlation between the Leeds assessment of neuropathic symptoms and signs pain scale and electromyographic findings. [Conclusion] We found that the severity of electrophysiologic findings of ulnar nerve entrapment at the elbow did not differ between neuropathic and non-neuropathic groups as assessed by the Leeds assessment of neuropathic symptoms and signs pain scale.

Key words: Ulnar nerve, Neuropatic pain
\end{abstract}

(This article was submitted Feb. 19, 2015, and was accepted Apr. 13, 2015)

\section{INTRODUCTION}

Ulnar nerve neuropathies are the second most commonly seen entrapment neuropathy of the upper extremities after carpal tunnel syndrome. Although the ulnar nerve is most commonly entrapped at the elbow, it can also be entrapped at the upper arm and forearm. The most important signs of ulnar neuropathy at the elbow are numbness of 4th and 5th digits, hypoesthesia of the medial palm, atrophy and paresthesia of ulnar nerve innervated hand muscles, and sometimes flexion deformity of fingers due to motor dysfunction of the flexor carpi ulnaris muscle.

Paresthesia may be increased by flexion posture or by pressing on the ulnar groove, and the ulnar nerve may be-

\section{*Corresponding author. Gulistan Halac (E-mail: halacdr@} yahoo.com)

(C2015 The Society of Physical Therapy Science. Published by IPEC Inc. This is an open-access article distributed under the terms of the Creative Commons Attribution Non-Commercial No Derivatives (by-ncnd) License $<$ http://creativecommons.org/licenses/by-nc-nd/3.0/> . come enlarged and palpable. When there is pain, it can be localized on the elbow or extend to the medial forearm or wrist. Pain in ulnar entrapment neuropathies can be due to either neuropathic mechanisms or mixed pain with additive musculoskeletal and joint nociceptive mechanisms. Understanding the mechanism of pain and planning the appropriate treatment plays an important role in improvement of the quality of life of the patients ${ }^{1)}$.

The LANSS (Leeds assessment of neuropathic symptoms and signs) pain scale was first performed by Bennett in order to clinically differentiate neuropathic pain from nociceptive pain $^{2}$. The time needed to perform this scale is short, and evaluation is easy. Turkish validation of the LANSS pain scale was performed by Yucel et al. in 2004 ${ }^{3}$. The total score of this test is 24 , and a total score of $\geq 12$ is considered to be related to a pain due to neuropathic mechanisms.

The results between of previous studies concerning the correlation of symptoms with the severity of electrophysiological findings among patients with ulnar neuropathy differ. There aren't any studies evaluating the correlation of electrophysiological findings with neuropathic pain as determined by the LANSS pain svale. In this study, we aimed 
to evaluate pain among ulnar neuropathy patients by the LANSS pain scale and to determine if it is correlated with the severity of electrophysiological findings.

\section{SUBJECTS AND METHODS}

Patients sent to an electrophysiology laboratory with suspected ulnar entrapment neuropathy were evaluated by history, systemic, and neurological examinations. The total number of patients was 63 . Only patients with ulnar entrapment neuropathy symptoms and additional sensory and motor deficits were included in the study (muscle weakness of ulnar innervated muscles, numbness of the fourth and fifth fingers, positive Tinnel's sign at the elbow, sensory deficit over ulnar nerve innervated skin). The patients had been complaining about these symptoms for at least 3 months. The exclusion criteria were clinical signs of polyneuropathy; cervical radiculopathy; brachial plexopathy; syringomyelia; amyotrophic lateral sclerosis (ALS); history of orthopedic and rheumatological diseases; hereditary pressuresensitive neuropathy; diabetes mellitus; renal failure; hypothyroidism; malignancy; history of alcohol, toxin, or drug usage; and history of trauma or surgery of the upper extremity and/ or neck area.

Electrophysiological evaluation of the study group was performed by an expert without knowledge of their symptoms or the results of a physical examination. Nerve conduction studies, needle electromyography, and the LANSS pain scale were performed in order by a Keypoint electromyography machine (Medtronic, Skovlunde, Denmark). Limb temperature of the patients was kept above $32{ }^{\circ} \mathrm{C}$. Bilateral ulnar motor distal latency; ulnar nerve motor conduction velocity at the wrist, below the elbow, and above the elbow segments; and bilateral ulnar sensory action potential latency, amplitude, and conduction velocity were studied in each patient. Also, median nerve motor distal latency, median nerve motor conduction velocity at the elbow segment, and median nerve sensory action potential latency, amplitude, and conduction velocity were studied in order to detect any median nerve involvement on the affected part.

Sensory nerve conduction studies: Sensory nerve conduction studies (NCs) were performed with an orthodromic technique that stimulated the ulnar nerve at digit V. Sensory nerve conduction potentials (SNAPs) were recorded by surface electrodes placed over the wrist at a distance of $11 \mathrm{~cm}$ from the active stimulating electrode. Stimulation of the sensory nerve was characterized by a duration of $100 \mu$ s and an intensity of 10-30 mA with a filter setting of $20 \mathrm{~Hz}$ to $2 \mathrm{kHz}$. Distal sensory latencies, sensory conduction velocities, and peak to peak sensory nerve action potential amplitudes were measured. NCs of the median nerve were performed with the same technique by stimulating the median nerve at digit II. SNAPs were recorded by surface electrodes placed over the wrist at a distance of $13 \mathrm{~cm}$ from the active stimulating electrode. Latency, amplitude, and sensory conduction velocities were recorded during sensory nerve conduction studies.

Motor nerve conduction studies: Stimulation of the motor nerve was characterized by a duration of $100 \mu$ s and an intensity of 30-90 mA with a band-pass filter setting of $20 \mathrm{~Hz}$ to $10 \mathrm{kHz}$. Ulnar nerve compound muscle action potential
(CMAP) was recorded by electrodes placed over the abductor digiti minimi and frontal dorsal interosseus muscles. The ulnar nerve was stimulated at the wrist $3 \mathrm{~cm}$ proximal to the ulnar prominence and $5 \mathrm{~cm}$ below and $5 \mathrm{~cm}$ above the epicondyle. Motor conduction studies were performed with the elbow of the study groups were lying 70 degrees from the horizontal position. Median nerve motor conduction studies were performed by placing the recording electrode over the abductor pollicis brevis (APB) muscle and providing stimulation from wrist and elbow. Active surface electrodes were placed over the APB muscle between the metacarpophalangeal joint of the thumb and distal wrist line while the reference electrode and ground electrode were placed over the proximal phalanx of the thumb and forearm flexor surface, respectively. Distal motor latency was recorded from the beginning of CMAP. Distal latency, motor nerve conduction velocity (MCV), and baseline-to-negative peak amplitude were recorded during motor nerve conduction studies.

Needle electromyography study: The ulnar nerve innervated abductor digiti minimi (ADM), flexor digitorumindicis (FDI), flexor carpi ulnaris (FCU), flexor digitorum profundus (FDP) muscles; median nerve innervated APB muscle; and radial nerve innervated extensor digitorumcommunis (EDC) muscle were studied. The parameters for the needle electromyography study were $200 \mathrm{pV}$ for sensitivity, $100 \mathrm{msec}-10 \mathrm{n}$ for analysis time, and $20 \mathrm{~Hz}$ to $10 \mathrm{kHz}$ for frequency criteria.

Electrophysiological inclusion criteria for acceptance of study findings as abnormal:

- Motor nerve conduction velocity $<50 \mathrm{~m} / \mathrm{sec}$ at the elbowsegment.

- $>20 \%$ decrease in BKAP amplitude at the elbow segment compared with the wrist.

- Ulnar nerve sensory conduction velocity $<50 \mathrm{~m} / \mathrm{sec}$

After completion of neurological examinations and electrophysiological studies, the patients were grouped according to classification based on clinical and electrophysiological descriptions of ulnar neuropathy at the elbow as normal (class 1), mild (class 2), moderate (class 3), and severe (class $4)^{4)}$ (Table 1).

After diagnosis of ulnar neuropathy at the elbow, all patients underwent the Turkish version of the LANSS pain scale. According to the LANSS pain scale, the patients were grouped into two groups as those who scored 12 or more points and those who scored less than 12 points.

Statistical analysis was performed with SPSS for Windows. Defining parameters are given as the mean \pm standard deviation and percentage. Comparison between two groups were performed with using Pearson's $\chi^{2}$ test.

\section{RESULTS}

Based on the inclusion and exclusion criteria, 34 of the 63 patients were included in the study; the other 29 patients were excluded. Fifteen $(44.1 \%)$ of the included patients were male, while the rest $19(55.9 \%)$ were female. The mean age of the patients was 42.18 (19-63). Padua elbow ulnar neuropathy (EUN) classification ${ }^{4}$ ) was used to evaluate the ulnar entrapment of the ulnar neuropathy patients. According to this classification system, 15 (44\%), 14 (41.2\%), 4 
Table 1. Padua classification of ulnar neuropathy at the elbow

1. EUN: Normal findings
2. Mild EUN: Slowed motor nerve conduction velocity and normal sensory action potential when measuring over the elbow
3. Moderate EUN: Slowed motor nerve conduction velocity and decreased amplitude of SNAP when measuring over the elbow
4. Severe EUN: Decreased motor nerve conduction velocity when measuring over the elbow plus absent ulnar SNAP when
measuring over the 5th digit wrist segment
5. Very severe EUN: Absent hypothenar motor and sensory responses

(11.8\%), and $1(2.9 \%)$ patient were classified as class-2, class-3, class-4, and class-5, respectively. No patient was included in class-1. According to the LANSS pain scale 24 patients $(70.6 \%)$ scored under 12 points. The number of patients who achieved more than 12 points was $10(29.4 \%)$. Groups were compared by using the $\chi^{2}$ test, and no difference was detected.

The mean age of the patients who did not have neuropathic pain according to the LANSS pain scale was 45.33 (21-63). Twelve $(50 \%)$ of these patients were male, and the other $12(50 \%)$ were female. Ten $(41.7 \%)$ patients in this group were classified as class-2 and the rest were classified as class-3 (12 patients $(50 \%))$, class-4 (1 patient $(4.2 \%))$, and class-5 (1 patient $(4.2 \%))$, respectively.

The mean age of the patients who had neuropathic pain according to the LANSS pain scale was 34.6 (19-56). This group consisted of $3(30 \%)$ males and $7(70 \%)$ females. Five $(50 \%)$ patients in this group were classified as class-2, and the remaining were classified as class-3 (2 patients $(20 \%))$ and class-4 (3 patients $(30 \%)$. There were no class- 1 or class-5 patients according to Padua EUN classification. There was no correlation between the LANSS pain scale and EMG findings ( $\mathrm{p}>0.05)$.

\section{DISCUSSION}

The ulnar nerve is comprised of the C 8 and $\mathrm{T} 1$ anterior root motor and $\mathrm{C} 8$ posterior root sensory fibers. It can receive some fibers from $\mathrm{C} 7$, too. The ulnar nerve passes through the brachial plexus via the inferior and medial trunci and leaves it at the proximal axilla. It passes over the lateral wall of the axilla and passes to the medial side of the arm. It enters into the ulnar groove behind the medial epicondyle. This groove is the most important entrapment region. The ulnar nerve does not branch at the elbow. It innervates two muscles on the forearm, the FCU and ulnar branch of the FDP. It has branches extending to the hand muscles and to the skin of the hand over the distal forearm and wrist.

Ulnar neuropathy at the elbow level is seen 3-8 times more often among males compared with females ${ }^{5}$. Contreras et al. showed that females have 2-19 times more fat at the elbow and they speculated that this could be protective against entrapment ${ }^{6}$. However, the coronoid tubercle is 1.5 times bigger in males, and this could have a potential compressive effect. Despite these data, most of our patients were female $(55.9 \%)$. We did not consider body mass index (BMI) of our patients during evaluation. A study that compared females with a $\mathrm{BMI} \leq 22$ and those with a $\mathrm{BMI}>22$ showed that those with lower BMis had more ulnar neuropa- thy at the elbow level ${ }^{5)}$. Subcutaneous fat tissue is protective against acute ulnar neuropathy among females and external compression seems to be a more important cause of ulnar neuropathy at the elbow level ${ }^{5,6}$ ). The reason for the high incidence of ulnar neuropathy at the elbow level among males is suggested to be the higher amount of muscle tissue and strength of the forearm ${ }^{7)}$. The basis of this hypothesis is increasing compression under the FCU aponeurosis caused by isometric compression of the $\mathrm{FCU}^{8)}$.

Repeated flexion and extension movements comprise an important part of entrapments at the elbow. The elbow is coming to flexion from extension positiondistance between medial epicondyle and olecranon is $5 \mathrm{~mm}$ increased in every 45 degrees angle ${ }^{8)}$. The shape of the cubital tunnel changes from a round shape to an oval shape. The height of the cubital tunnel also decreases, and this causes a 55\% decrease in channel volume, resulting in increased pressure. The pressure over the ulnar nerve during extension is $7 \mathrm{mmHg}$, and with elbow flexion and changing of the position of the shoulder and elbow, this pressure increases to $11-24 \mathrm{mmHg}$. It has been shown that FCU contraction with elbow flexion results in $200 \mathrm{mmHg}$ of pressure over the ulnar nerve. Since the ulnar nerve passes behind the elbow rotation axis, traction and excursion also occurs and this causes 5-8 mm elongation of the nerve ${ }^{9)}$. Studies have shown that, the combination of shoulder abduction, elbow flexion and wrist extension at the same time can increase cubital tunnel pressure by 6 times and that the highest amount of pressure increase occurs with this position ${ }^{9,10)}$. During flexions that exceed 90 degrees, the intraneural pressure is more than the extraneural pressure. Our study was performed with the elbow lying in a position 70 degrees from the horizontal axis.

Patients with ulnar nerve entrapment neuropathy of the elbow require attention and are investigated because of pain. Early diagnosis, correct classification and understanding of the mechanisms underlying pain are needed to decide the appropriate treatment ${ }^{11)}$. The International Association for the Study of Pain (IASP) defines neuropathic pain as a pain induced by dysfunction in or damage to the nervous system $^{12)}$. Peripheric neuropathic pain is experienced due to the peripheric nervous system, and central neuropathic pain is experienced due to central nervous system damage. The symptoms of neuropathic pain are burning, a tingling sensation, and sometimes pain similar to an electric shock. Paresthesia-dysesthesia, allodynia-hyperalgesia, involuntary movements of the extremities, and autonomic system findings (sweating-vasomotor dysregulation and changes) are also observed. The Turkish version of the LANSS pain scale has been validated for differentiation of nociceptive 
pain from neuropathic pain ${ }^{3)}$. In this investigation, pain was evaluated via five questions about pain such as dysesthesia, autonomic dysfunction, induction of pain, paroxysmal pain, and thermal pain. Also, two neurological findings of neuropathic pain, allodynia and pinprick threshold, were examined.

Truni et al. found that severity of carpal tunnel syndrome symptoms did not differ between neuropathic and nonneuropathic groups ${ }^{13}$. In our study, the number of cases in which neuropathic mechanisms could be the underlying cause of pain due to ulnar neuropathy at the elbow was 10 (29.4\%). The number of cases that did not support neuropathic pain was $24(70.6 \%)$. We could not compare these two groups, since few cases were experiencing pain due to neuropathic mechanisms. We found that electrophysiologic findings of the severity of ulnar nerve entrapment at the elbow did not differ between neuropathic and non-neuropathic groups as assessed by the LANNS pain scale. We did not have clinically class- 1 ulnar neuropathic patients. This was due to our small study group. Also, all the patients included in our study were patients that had been sent to an electroneurophysiology laboratory, and this can be the reason for the absence of early stage patients.

In conclusion, one point that we should remember is that, during routine $\mathrm{EMG}$, nerves with middle and large diameters are investigated. $\mathrm{C}$ and $\mathrm{A}$ delta fiber activities, which play an important role in pain conduction, cannot be examined during a routine EMG investigation. In order to investigate the activity of these fibers, special investigations such as microneurography and laser evoked potentials are needed. In our study, we used nerve conduction and needle EMG studies. We performed this study because there was no report about this topic in the literature and performed it as a step to further studies. We believe that use of large groups of subjects and detailed electrophysiological investigations will give rise to detailed data.

\section{REFERENCES}

1) Ha M, Son Y, Han D: Effect of median nerve mobilization and median nerve self-mobilization on median motor nerve conduction velocity. J Phy Ther Sci, 2012, 24: 801-804. [CrossRef]

2) Bennett M: The LANSS Pain Scale: the Leeds assessment of neuropathic symptoms and signs. Pain, 2001, 92: 147-157. [Medline] [CrossRef]

3) Yücel A, Şenocak M, Kocasoy Orhan E, et al.: Results of the Leeds assessment of neuropathic symptoms and signs pain scale in Turkey: a validation study. J Pain, 2004, 5: 427-432. [Medline] [CrossRef]

4) Padua L, Aprile I, Mazza O, et al.: Neurophysiological classification of ulnar entrapment across the elbow. Neurol Sci, 2001, 22: 11-16. [Medline] [CrossRef]

5) Richardson JK, Green DF, Jamieson SC, et al.: Gender, body mass and age as risk factors for ulnar mononeuropathy at the elbow. Muscle Nerve, 2001, 24: 551-554. [Medline] [CrossRef]

6) Contreras MG, Warner MA, Charboneau WJ, et al.: Anatomy of the ulnar nerve at the elbow: potential relationship of acute ulnar neuropathy to gender differences. Clin Anat, 1998, 11: 372-378. [Medline] [CrossRef]

7) Barnekow-Bergkvist M, Hedberg G, Janlert U, et al.: Development of muscular endurance and strength from adolescence to adulthood and level of physical capacity in men and women at the age of 34 years. Scand J Med Sci Sports, 1996, 6: 145-155. [Medline] [CrossRef]

8) Werner CO, Ohlin P, Elmqvist D: Pressures recorded in ulnar neuropathy. Acta Orthop Scand, 1985, 56: 404-406. [Medline] [CrossRef]

9) Bozentka DJ: Cubital tunnel syndrome pathophysiology. Clin Orthop Relat Res, 1998, (351): 90-94. [Medline]

10) Green JR Jr, Rayan GM: The cubital tunnel: anatomic, histologic, and biomechanical study. J Shoulder Elbow Surg, 1999, 8: 466-470. [Medline] [CrossRef]

11) Kang JH, Lee YS: Sensory nerve conduction studies in the diagnosis of diabetic sensorimotor polyneuropathy: electrophysiological features. J Phys Ther Sci, 2012, 24: 139-142. [CrossRef]

12) Merskey H, Bogduk N: Classifications of chronic pain: Description of chronic pain syndromes and definition of pain terms. Report by the International Association for the Study of Pain Task Force on Taxonomy. In: Merskey H, Bogduk N (eds.) Seattle: IASP Press, 1994.

13) Truini A, Padua L, Biasiotta A, et al.: Differential involvement of A-delta and A-beta fibres in neuropathic pain related to carpal tunnel syndrome. Pain, 2009, 145: 105-109. [Medline] [CrossRef] 